\title{
Chest X-rays findings in COVID 19 patients at a University Teaching Hospital - A descriptive study

\author{
Misbah Durrani' ${ }^{1}$ Inam ul Haq \\ Ume Kalsoom ${ }^{3}$, Anum Yousaf ${ }^{4}$
}

\begin{abstract}
Objective: To analyze Chest X-ray findings in COVID 19 positive patients, presented at corona filtration center, Benazir Bhutto Hospital Rawalpindi, based on CXR classification of British Society of Thoracic Imaging (BSTI).

Methods: In this study, all RT-PCR COVID-19 positive patients screened at corona filtration center, Benazir Bhutto hospital Rawalpindi from $20^{\text {th }}$ March 2020 to $10^{\text {th }}$ April 2020 were included. Mean age of the cohort with age range was calculated. Presenting complaints \& Co-morbid were analyzed and tabulated in frequencies and percentages. Portable CXR findings were classified according to BSTI classification and documented in frequencies and percentages.

Results: Mean age of the patients was 44 years. Presenting complaints were cough $20(67 \%)$, fever 18 $(60 \%)$, shortness of breath $11(37 \%)$, sore throat six (20\%), loss of sense of taste and smell four(13\%). Main co-morbid was hypertension six (20\%). Two (7\%) patients had normal and seven $(23 \%)$ had classical COVID CXRs. $21(70 \%)$ patients were in indeterminate group with only one (3\%) having unilateral lung disease. Three (10\%) patients had diffuse lung involvement and $18(60 \%)$ had peripheral lung involvement. Majority of patients $19(63 \%)$, had bilateral middle and lower zonal involvement.

Conclusions: In this study, COVID-19 CXRs generally manifested a spectrum of pure ground glass, mixed ground glass opacities to consolidation in bilateral peripheral middle and lower lung zones. BSTI CXR reporting classification of COVID-19 is valid in our patients with addition of middle zonal involvement in classical COVID-19 criteria as opposed to just lower zone involvement.
\end{abstract}

KEYWORDS: COVID-19 patients, Chest X-rays (CXR), Corona virus, British Society of thoracic Imaging classification (BSTI).

doi: https://doi.org/10.12669/pjms.36.COVID19-S4.2778

How to cite this:

Durrani M, Inam-ul-Haq, Kalsoom U, Yousaf A. Chest X-rays findings in COVID 19 patients at a University Teaching Hospital - A descriptive study. Pak J Med Sci. 2020;36(COVID19-S4):COVID19-S22-S26. doi: https://doi.org/10.12669/pjms.36.COVID19-S4.2778

This is an Open Access article distributed under the terms of the Creative Commons Attribution License (http://creativecommons.org/licenses/by/3.0), which permits unrestricted use, distribution, and reproduction in any medium, provided the original work is properly cited.

1. Dr. Misbah Durrani, MCPS. FCPS

2. Dr. Inam ul Haq, FCPS. MHPE.

Al-Shifa Trust Eye Hospital, Rawalpindi, Pakistan.

3. Dr. Ume Kalsoom FCPS.

4. Dr. Anum Yousaf, Resident.

1,3,4: Department of Diagnostic Radiology. Benazir Bhutto Hospital, Rawalpindi, Pakistan.

Correspondence:

Dr. Misbah Durrani, FCPS. Assistant Professor,

Rawalpindi Medical University,

Visiting Radiologist, Benazir Bhutto Hospital, Rawalpindi, Pakistan.

E-mail: misbahdurrani73@gmail.com

* Received for Publication:

April 25, 2020

* Revision Received:

* Revision Accepted:

\section{INTRODUCTION}

Devastations of COVID 19 are revealing day by day. This pandemic has spread all across the globe. This disease is still a mystery. We are not able to comprehend; why this disease has different out comes in different individuals irrespective of age, race and regional constraints. Spectrum of COVID 19 positive patients varies from asymptomatic to patient on ventilators and ultimately death.

Radiological evaluation is one way of looking in the body. ${ }^{1}$ CXRs are the most commonly performed investigation in COVID 19 suspected cases. ${ }^{2}$ British Society of Thoracic Imaging (BSTI) has classified CXRs findings, based on European patients. ${ }^{3}$ Our 
local population is different both in habitat and disease patterns, it was therefore thought to see pattern of CXR findings in our COVID 19 positive patients. CT scan is a preferred investigation in terms of diagnosis and disease follow up, but it is not feasible to use it as a screening tool in terms of its availability and rigorous time consuming decontamination measures.

The purpose of this study was to analyze chest $\mathrm{X}$-rays findings in our patients based on British Society of Thoracic Imaging classification and to evaluate disease pattern in terms of any deviation or similarity. Another rationale is that X-ray facilities are available in basic health units and this study will enhance our clinicians understanding of CXR findings in suspected COVID 19 patients.

\section{METHODS}

This is a retrospective descriptive study conducted at Isolation ward $\mathrm{BBH}$ hospital Rawalpindi. All RT-PCR COVID 19 positive patients presented in corona filtration center Benazir Bhutto hospital from 20 March 2020 to 10 April 2020 were included. Benazir Bhutto hospital Rawalpindi is a teaching hospital affiliated with Rawalpindi Medical University". It has a designated COVID-19 filter clinic where suspected patients are screened initially with portable chest $\mathrm{X}$ - rays and RT-PCR throat swab. Chest X-rays are stored in central computer server of Benazir Bhutto hospital. Chest radiographs of all COVID-19 confirmed patients from 20th March 2020 to 10th April 2020 were included in this study, irrespective of age or gender and were classified according to BSTI classification.

Data Analysis and results: Quantitative variables like age is presented as mean along with age range. Qualitative variables like gender and co morbid were presented as frequency and percentages. Outcome variable, portable CXR findings were presented as frequency and percentages.

\section{RESULTS}

Thirty COVID 19 positive cases had reported during the specified time, all were included in the study. Mean age of the patients was 44 with age range 7- 81 years. Two patients were of seven years of age. There were $24(80 \%)$ males and six $(20 \%)$ females. Male predilection of this disease is noted down in this study. 10 (33\%) patients had history of travel. History of contact was positive in only five (17\%) patients. Cough was the predominant presenting complaint in
20 (67\% patients, followed by fever in $18(60 \%)$, Shortness of breath $11(37 \%)$, sore throat six $(20 \%)$, loss of sense of smell and taste four (13\%) and GIT complaints in three $(10 \%)$ patients. In eight $(27 \%)$ patients there were no co morbid. Six $(20 \%)$ patients had ischemic heart disease and hypertension, three $(10 \%)$ patients had diabetes, two $(7 \%)$ patients were smoker, and six $(18 \%)$ patients had other diseases tabulated in Table-I.

Chest X-rays of all thirty patients were classified as normal, classical and indeterminate according to BSTI COVID-19 CXR classification. Two patients had normal chest X-rays (7\%) and seven patients $(23 \%)$ had classical picture of bilateral

Table-I: Patient's profile.

\begin{tabular}{|c|c|c|c|}
\hline S. No & & & Findings \\
\hline \multirow{2}{*}{1} & \multirow{2}{*}{ Age } & Mean & 44 years \\
\hline & & Range & 7-81 years \\
\hline \multirow{3}{*}{2} & \multirow{3}{*}{ Gender } & Mll & $n(\%)$ \\
\hline & & Miare & $24(80 \%)$ \\
\hline & & Female & $6(20 \%)$ \\
\hline 3 & H/O Travel & & $10(33 \%)$ \\
\hline 4 & $\mathrm{H} / \mathrm{O}$ Contact & & $5(17 \%)$ \\
\hline \multirow{6}{*}{5} & \multirow{6}{*}{ Symptoms } & Cough & $20(67 \%)$ \\
\hline & & Fever & $18(60 \%)$ \\
\hline & & Shortness of breath & $11(37 \%)$ \\
\hline & & Sore throat & $6(20 \%)$ \\
\hline & & $\begin{array}{l}\text { Loss of sense of } \\
\text { taste and smell }\end{array}$ & $4(13 \%)$ \\
\hline & & GIT symptoms & $3(10 \%)$ \\
\hline \multirow{10}{*}{6} & \multirow{10}{*}{ Co-morbid } & No co-morbid & $8(27 \%)$ \\
\hline & & $\begin{array}{l}\text { IHD \& } \\
\text { Hypertension }\end{array}$ & $6(20 \%)$ \\
\hline & & Diabetes Mellitus & $3(10 \%)$ \\
\hline & & Smoker & $2(7 \%)$ \\
\hline & & Renal complaints & $1(3 \%)$ \\
\hline & & stroke & $1(3 \%)$ \\
\hline & & Tuberculosis & $1(3 \%)$ \\
\hline & & Asthma & $1(3 \%)$ \\
\hline & & Arthritis & $1(3 \%)$ \\
\hline & & Malignancies & $1(3 \%)$ \\
\hline
\end{tabular}

(Figures are presented as whole numbers with percentages in brackets). 
peripheral, basal ground glass haze/consolidation. Rests of twenty-one patients $(70 \%)$ were falling in indeterminate group with one $(3 \%)$ having unilateral lung disease and $20(67 \%)$ patients had bilateral lung disease. Diffuse lung involvement was seen in three $(10 \%)$ and peripheral lung involvement in $18(60 \%)$ of patients. Majority of indeterminate patients, 19 (63\%) had bilateral middle and lower zonal involvement and only two $(7 \%)$ patients had middle zone involvement. Associated features in indeterminate group were pleural effusion four $(13 \%)$, old healed calcific granulomas one $(3 \%)$, and bilateral hilar lymphadenopathy one (3\%). There were no cavitating lesions or pneumothorax. Table-II

\section{DISCUSSION}

Viruses belonging to the family of coronavirdae had already resulted in acute respiratory distress

Table-II: Radiographic findings using BSTI COVID-19 CXR report proforma in patients.

\begin{tabular}{lc}
\hline Findings & No of patients \\
\hline 1. NORMAL correlated with RT-PCR & $2(7 \%)$ \\
2. CLASSIC / PROBABLE COVID -19 & \\
Consolidation / ground glass haze & \\
- Bilateral, peripheral ,basal & $7(23 \%)$ \\
Indeterminate for COVID -19 & \\
- Consolidation/ground glass haze & \\
i). Location & $1(3 \%)$ \\
Unilateral & $20(67 \%)$ \\
Bilateral & \\
ii). Distribution & $3(10 \%)$ \\
Diffuse lung involvement & $18(60 \%)$ \\
Peripheral lung involvement & \\
iii). Zonal predominance & $19(63 \%)$ \\
Middle and lower zones involvement & $2(7 \%)$ \\
Only Middle zones involvement & $1(3 \%)$ \\
- Associated Features & \\
Pleural Effusion & \\
Old healed calcific granulomas & \\
Cavitating lesions/pneumothorax & \\
Bilateral hilar lymphadenopathy & \\
\hline & \\
Figures ar & \\
\hline
\end{tabular}

(Figures are presented as whole numbers with percentages in brackets). syndrome (SARS) in 2003 and Middle East respiratory syndrome ${ }^{4,5}$ (MERS) in 2012. COVID 19 virus has recently erupted and is still a mystery. Lot of research is going on all across the world and knowledge is being shared. Portable chest X-ray is the most commonly performed radiological investigation in terms of feasibility and cost effectiveness even in developed countries. In a dedicated corona filtration centre like Benazir Bhutto hospital Rawalpindi where on an average more than hundred suspected patients are being screened for COVID 19, portable chest X-ray is the optimal radiological screening tool. Strict decontamination measures could be ensured which are not possible in busy general OPD X-ray rooms. Due to limited RT-PCR kits and delayed results up to 48 hours, cases of high clinical suspicion with positive CXR findings are kept in isolation wards. CXR has a low sensitivity ${ }^{6}$ and it is difficult to distinguish between COVID 19 and other viral pneumonias purely on CXR findings. CT scan is the preferred imaging modality regarding early detection of disease as well as of its complications but it has infection control challenges including strict decontamination measures, ventilation and airflow. ${ }^{7}$ In HY Yoon et al study, ${ }^{8} 33 \%$ patients had abnormal initial radiographic findings in contrast to $93 \%$ abnormal chest findings in our study. In SARS $^{9}$ these initial abnormal chest findings were in 78.3-82.4\% and in MERS ${ }^{10}$ 83.6\%. In Wong HYF et al. ${ }^{11}$ study consolidation was found in $47 \%$ of cases and this finding is consistent with other studies. ${ }^{12}$

There is no published study in literature as yet regarding COVID-19 CXR findings in Pakistan, however there are lots of studies on Chinese, Korean, American and European population. British society of thoracic imaging ${ }^{13}$ has classified COVID -19 chest X-rays as normal co related with RT-PCR, ${ }^{14}$ classical, having multiple bilateral, peripheral basal opacities more bilateral than unilateral, indeterminate that does not fit into classical or non COVID descriptors and Non COVID-19 X-rays having pneumothorax, pleural effusion and pulmonary edema. UCLA CXR COVID reporting classification is based upon recommendation from Radiological society of North America ${ }^{15}$ as typical having multifocal peripheral opacities with differential diagnosis of drug toxicity, influenza pneumonia and organizational pneumonia, indeterminate as non peripheral consolidation with differential of lots of infectious processes, atypical with uncommon imaging features and negative (does not exclude COVID-19). 


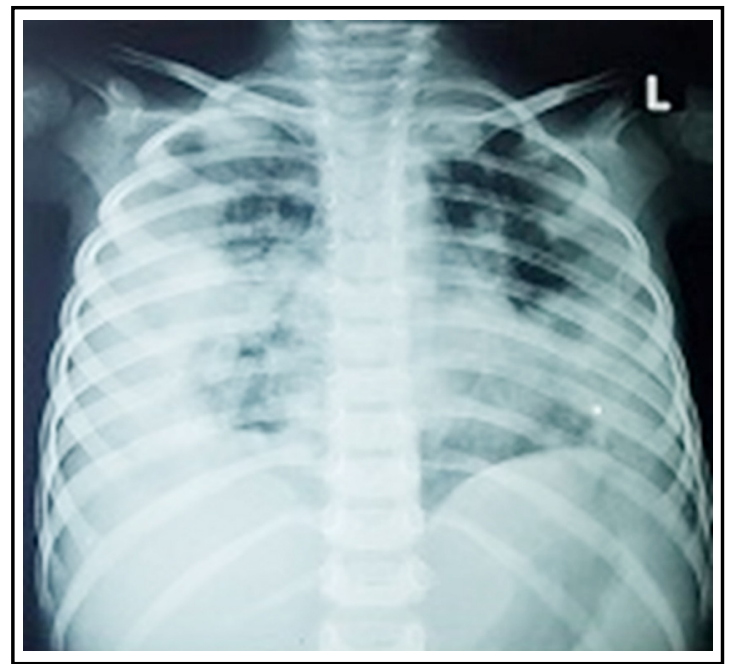

Fig.1: Chest X-ray of a seven years old female child showing Extensive Bilateral peripheral consolidation with air bronchograms predominantly right side, partially obscured right diaphragmatic silhouette and obscured ipsilateral CP angle. Radiographic findings are indeterminate for COVID-19. She had strong contact history, presented in ER with acute complains of SOB, cough, fever and flu.

Radiological findings were described according to Fleischner Society glossary of terms for Thoracic imaging. ${ }^{16}$ Ground glass opacities were defined as increased opacification of lung parenchyma not obscuring blood vessels and bronchi. Consolidation was described as homogenous opacification of lung parenchyma obscuring blood vessels and bronchi. We classified all CXRs on BSTI classification and found that majority of patients had bilateral, peripheral ground glass opacities and consolidation as documented in international studies. There may be diffuse lung involvement with perihilar infiltrates as well, marking severity of disease process. Fig.1.

Our study also shows that only seven (23\%) of patients had BSTI classical picture of COVID -19 pneumonia of bilateral peripheral basal consolidation /ground glass haze. Majority of patients were of indeterminate group because of bilateral peripheral, multifocal middle and lower zonal lung involvement. Fig.2. This can imply that radiographic presentation of our patients was more severe in intensity. ${ }^{17}$

This pattern of consolidation had variable presentation in terms of shape and density. Some patients had smooth homogenous consolidation, while majority had inhomogenous, confluencing or patchy nodular opacities in peripheral distribution. Three patients with diffuse lung involvement had severe disease with no cephalocaudal or peripheral

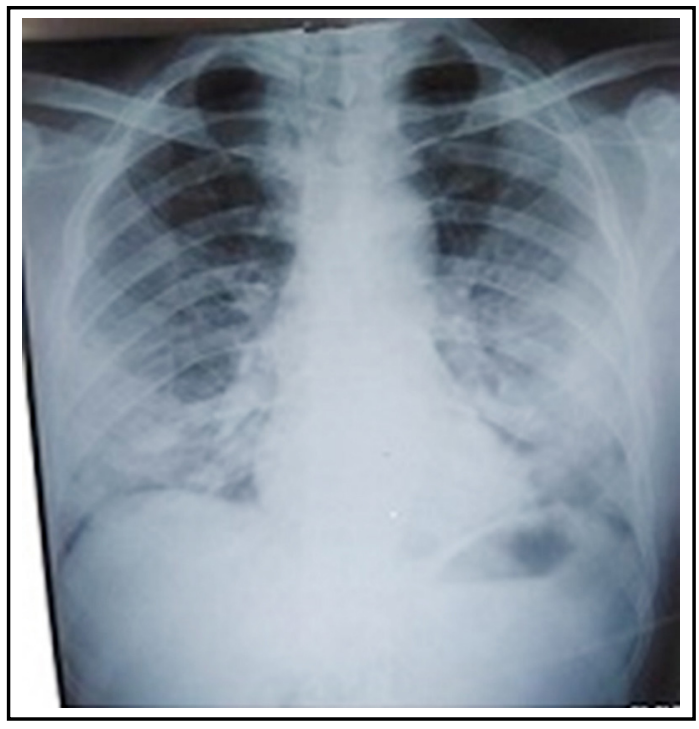

Fig.2: Chest $X$-ray image of a COVID -19 positive 56 years old male with no travel/contact history, presented with high grade fever, sore throat and cough for two days, showing bilateral mid and lower zones homogenous consolidation in peripheral distribution $(\mathrm{L}>\mathrm{R})$ along with Obscuration of both $\mathrm{CP}$ angles- Findings fall in the category of indeterminate for COVID-19.

versus central discernment. Inclusion of middle lobe involvement in classical CXR definition of COVID -19 should be considered for Pakistani population. This could be a roadmap for future studies on a larger scale. There was no literature available from our neighboring countries to see if there was any similarity in classical CXR presentation of COVID -19 in South Asian population.

Indeterminate group included radiological characterization of COVID-19 keeping in view, peculiar presence of tuberculosis, seasonal emergence of allergic chest diseases and hypersensitivity pneumonitis in our local population. Pulmonary edema, interstitial pneumonitis and drug induced pneumonitis in immune-compromised patients can also mimic COVID-19 pneumonia. Uncommon imaging features such as lymph adenopathy and pleural effusion as mentioned in international studies were also uncommon in our local population.

Limitations of the study: It included small sample size of just thirty patients as they were collected in initial twenty days of pandemic in our country. Now with increasing number of COVID positive patients all over Pakistan, a more comprehensive study should follow. Novelty of this early study of CXR finding of COVID-19 in Pakistan outweighed this limitation. Another limitation was absence of 
serial CXRs to see progression of disease, variable presentations and long term outcomes in our population. Clinical significance of this study is to provide an early insight into CXR finding of our patients. Portable chest $\mathrm{X}$-rays ${ }^{18}$ are most readily available and feasible investigation in our urban and rural set ups. Its additional advantage is that there are minimal chances of cross contamination. Therefore, our general practitioners, clinicians, radiologists and paramedical health care workers can all benefit from findings of this study.

\section{CONCLUSION}

COVID-19 pneumonia generally manifested a spectrum of pure ground glass, mixed ground glass opacities to consolidation in bilateral peripheral middle and lower lung zones in our local population. BSTI chest reporting classification COVID-19 is valid in our patients with addition of middle zonal involvement in classical COVID-19 criteria as opposed to just lower zone involvement.

Acknowledgements: Vice chancellor RMU Professor M. Umer, Medical superintendent BBH Dr. Rafiq Ahmed, Dr. Nasir khan HOD radiology Holy family hospital, DMS admin BBH Dr Inayat ur Rehman for their support and facilitation in collection of data.

Conflict of Interest: The authors have no potential conflict of interest to disclose.

\section{Grant Support \& Financial Disclosures: None.}

\section{REFERENCES}

1. British Society of Thoracic Imaging. Background COVID-19. First cases Wuhan City China. 2020;(March):28

2. Jacobi A, Chung M, Bernheim A, Eber C. Portable chest X-ray in coronavirus disease-19 (COVID-19): A pictorial review. Clin Imaging. 2020;64:35-42. doi: 10.1016/j. clinimag.2020.04.001

3. Shi H, Han X, Jiang N, Cao Y, Alwalid O, Gu J, et al. Radiological findings from 81 patients with COVID-19 pneumonia in Wuhan, China: a descriptive study. Lancet Infect Dis. 2020;20(4):425-434. doi: 10.1016/S14733099(20)30086-4

4. Ajlan AM, Ahyad RA, Jamjoom LG, Alharthy A, Madani TA. Middle East respiratory syndrome coronavirus (MERS$\mathrm{CoV})$ infection: Chest CT findings. Am J Roentgenol. 2014;203(4):782-787.

5. Choi WJ, Lee KN, Kang EJ, Lee H. Middle east respiratory syndrome-coronavirus infection: A case report of serial computed tomographic findings in a young male patient. Korean J Radiol. 2016;17(1):166-170.
6. Zhou Z, Guo D, Li C, Fang Z, Chen L, Yang R, et al. Coronavirus disease 2019: initial chest CT findings. Eur Radiol. 2020;2 (December 2019).

7. Strunk JL, Temesgen H, Andersen H, Packalen P. Imaging Profile of the COVID-19 Infection: Radiologic Findings and Literature Review Authors: 2014;80(2):1-8.

8. Yoon SH, Lee KH, Kim JY, Lee YK, Ko H, Kim KH, et al. Chest radiographic and CT findings of the 2019 novel coronavirus disease (Covid-19): Analysis of nine patients treated in Korea. Korean J Radiol. 2020;21(4):498-504.

9. Antonio GE, Ooi CGC, Wong KT, Tsui ELH, Wong JSW, Sy ANL, et al. Radiographic-clinical correlation in severe acute respiratory syndrome: Study of 1373 patients in Hong Kong. Radiology. 2005;237(3):1081-1090.

10. Das KM, Lee EY, Al Jawder SE, Enani MA, Singh R, Skakni L, et al. Acute middle east respiratory syndrome coronavirus: Temporal lung changes observed on the chest radiographs of 55 patients. Am J Roentgenol. 2015;205(3):W267-W274.

11. Wong HYF, Lam HYS, Fong AH-T, Leung ST, Chin TWY, Lo CSY, et al. Frequency and Distribution of Chest Radiographic Findings in COVID-19 Positive Patients. Radiology. 2019;201160.

12. Strunk JL, Temesgen H, Andersen H, Packalen P. In Press Ines. 2014;80(2):1-8.

13. Rodrigues JCL, Hare SS, Edey A, Devaraj A, Jacob J, Johnstone A, et al. An update on COVID-19 for the radiologist - A British society of Thoracic Imaging statement. Clin Radiol. 2020;75(5):323-325.

14. Ai T, Yang Z, Xia L. Correlation of Chest CT and RT-PCR Testing in Coronavirus Disease. Radiology. 2020;2019:1-8.

15.. Simpson S, Kay FU, Abbara S, Bhalla S, Chung JH, Chung $\mathrm{M}$, et al. Radiological Society of North America Expert Consensus Statement on Reporting Chest CT Findings Related to COVID-19. Endorsed by the Society of Thoracic Radiology, the American College of Radiology, and RSNA. Radiol Cardiothorac Imaging. 2020;2(2):e200152.

16. Hansell DM, Bankier AA, MacMahon H, McLoud TC, Müller NL, Remy J. Fleischner Society: Glossary of terms for thoracic imaging. Radiology. 2008;246(3):697-722.

17. Zhu N, Zhang D, Wang W, Li X, Yang B, Song J, et al. A novel coronavirus from patients with pneumonia in China, 2019. N Engl J Med. 2020;382(8):727-733.

18. Zu ZY, Jiang M Di, Xu PP, Chen W, Ni QQ, Lu GM, et al. Coronavirus Disease 2019 (COVID-19): A Perspective from China. Radiology. 2020;2019:200490.

\section{Authors' Contribution:}

MD: Conceived, designed, manuscript writing and final review.

UK \& AY: Did data collection and manuscript writing.

IUH: Did statistical analysis and editing of manuscript. 